\title{
e-Migrinter
}

$11 \mid 2013$

Et l'immobilité dans la circulation?

\section{La figure de Pénélope ou l'immobilité dans le contexte des migrations circulaires}

\section{Florence Boyer}

\section{(2) OpenEdition}

\section{Journals}

Édition électronique

URL : https://journals.openedition.org/e-migrinter/229

DOI : 10.4000/e-migrinter.229

ISSN : 1961-9685

Éditeur

UMR 7301 - Migrinter

Édition imprimée

Date de publication : 1 septembre 2013

Pagination : 53-65

ISSN : 1961-9685

\section{Référence électronique}

Florence Boyer, «La figure de Pénélope ou l'immobilité dans le contexte des migrations circulaires », eMigrinter [En ligne], 11 | 2013, mis en ligne le, consulté le 20 mai 2021. URL : http://

journals.openedition.org/e-migrinter/229; DOI : https://doi.org/10.4000/e-migrinter.229

Tous droits réservés 


\section{La figure de Pénélope ou l'immobilité dans le contexte des migrations circulaires}

Florence Boyer

Photographie $\mathbf{n}^{\circ} \mathbf{1}$ : Battage du mil par des femmes en l'absence de leurs époux.

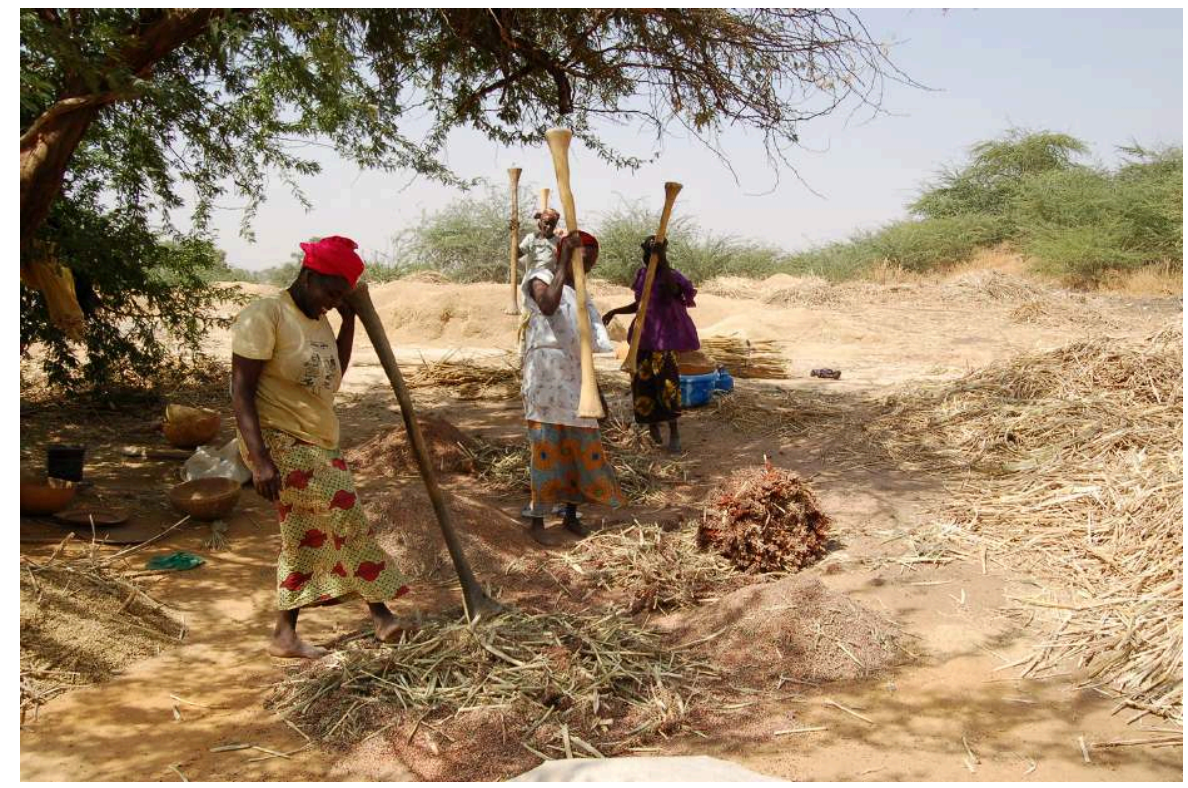

Source : Florence Boyer.

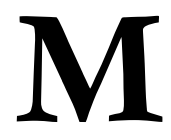

obilité et immobilité peuvent s'envisager comme les deux facettes d'un même questionnement, à savoir le positionnement de l'individu dans l'espace et dans le temps. Un individu est qualifié de mobile en regard d'autres qui sont qualifiés d'immobiles, et inversement.

Cependant, cette situation d'immobilité n'est que rarement interrogée en tant que telle contrairement à celle de mobilité -, de même que les relations, les liens entre ces deux situations. Un autre paradoxe tient au fait que la mobilité est observée alors que les groupes, les individus sont en situation d'immobilité ou d'installation.
Interroger ces deux facettes que sont la mobilité et l'immobilité amène à observer différentes échelles spatiales et temporelles. De même que la "mobilité généralisée ", expression de plus en plus présente dans la littérature scientifique, n'a pas vraiment de réalité, "l'immobilité généralisée » est très difficilement observable. Ainsi, dans des contextes de migrations internationales, on qualifiera d'immobile celui qui ne pratique pas une telle mobilité ; dans des contextes de multirésidence, est immobile celui qui n'est attaché qu'à un seul logement. L'immobilité se définit ainsi en regard d'une forme particulière de mobilité; les mobilités quotidiennes étant sans doute la forme la plus largement partagée.

Si la mobilité des uns permet l'immobilité des autres, et inversement, ces pratiques spatiales constituent des enjeux en termes de choix individuel, de négociation au sein des groupes familiaux. Les hiérarchies, les statuts sociaux, les rapports 
de genre, de classes d'âge ou le capital des individus sont autant d'éléments qui entrent en compte dans les stratégies de choix et de négociation; l'importance des uns et des autres pouvant varier en fonction du type de mobilité observé. Un autre élément qui peut intervenir dans cette réflexion sur les liens et les articulations entre mobilité et immobilité est la place de cette catégorie qu'est l'installation. Dans un sens commun, s'installer quelque part signifie avoir connu une mobilité, à laquelle il est a priori mis fin, par l'installation, et qui renvoie en même temps à un retour à une situation d'immobilité. Mobilité et immobilité sont à envisager comme des phases, des moments de la vie d'un individu, le terme de moment renvoyant autant à une échelle temporelle biographique que quotidienne, suivant le type de mobilité envisagé.

Nous analyserons les liens entre mobilité et immobilité au travers d'un cas particulier et en partant d'une forme de mobilité spécifique. L'immobilité permise par les mobilités, et inversement, s'illustre ainsi au travers du cas des migrations circulaires de Nigériens de la région de
Tahoua (centre du Niger) ; en effet, le départ des jeunes hommes en migration internationale et leur retour systématique après un temps d'absence variable, autorisent le maintien de leurs familles femmes, enfants, parents plus âgés - dans les villages de départ. Non seulement ils bénéficient des ressources de la migration, mais les circulations permettent d'éviter aussi une dispersion plus large et durable du groupe et par conséquent sa possible mise en péril. Pratiquées dans la plus grande partie du Sahel depuis quasiment un siècle, ces migrations circulaires ont connu des dynamiques, dépendantes autant des lieux de départ que des lieux d'arrivée. Que peut apporter une approche en termes de mobilité et d'immobilité à la compréhension des changements : changements des lieux et des rythmes de mobilité ? Comment interroger l'immobilité alors que les interrogations portent sur les migrations? En quoi adopter le point de vue des immobiles permet de dépasser une analyse sur les seuls liens ou complémentarités entre migrations et immobilité ?

Figure $\mathrm{n}^{\circ} 1$ : Migrations circulaires et situation de résidence : éléments de définition.

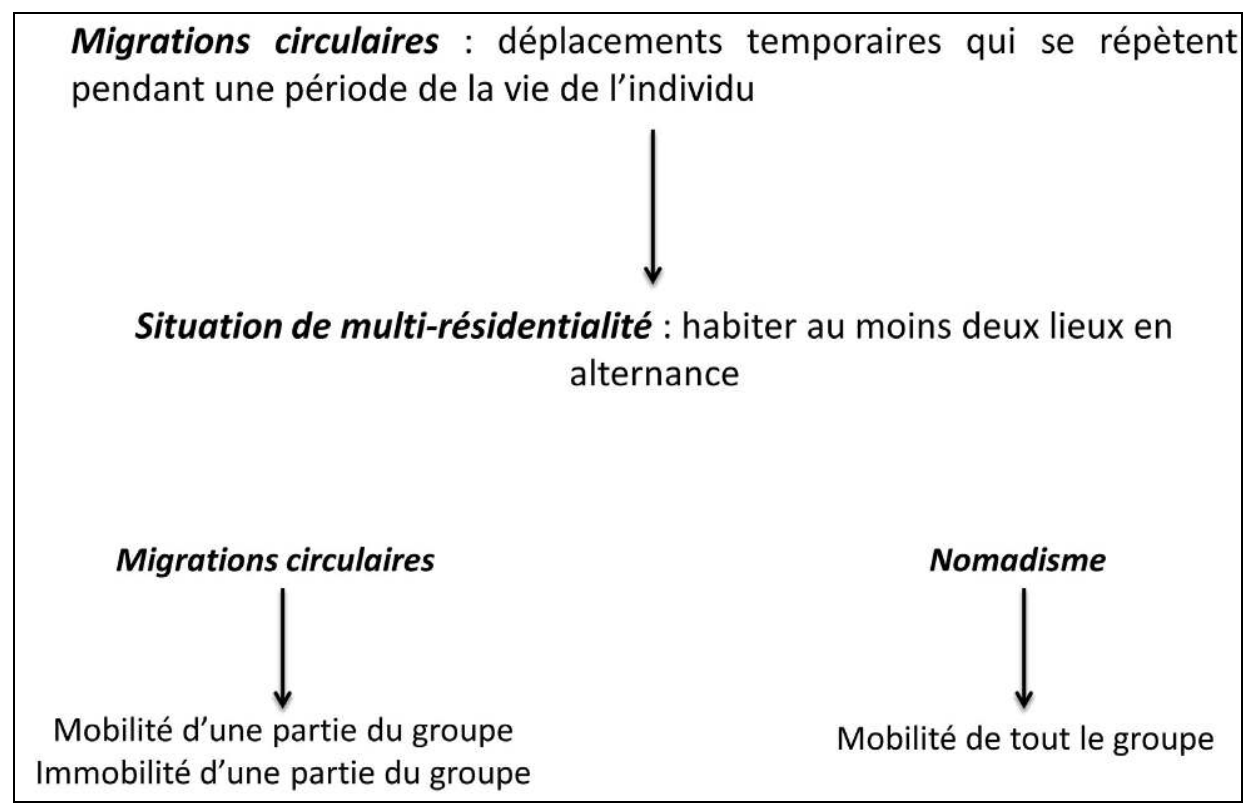

Source : Florence Boyer. 


\section{La migration des hommes face à l'immobilité des femmes}

Forme particulière de mobilité, les migrations circulaires se définissent d'une part par le caractère temporaire $\mathrm{du}$ mouvement et de l'absence, et d'autre part par la répétition de ce mouvement et de cette absence (Dureau, Florez, 2000). Alors que la migration temporaire n'implique qu'un déplacement, la migration circulaire repose sur la répétition du déplacement et par conséquent sur la pratique de divers lieux de résidence. La multi-résidence, sousjacente à cette notion de migration circulaire, suppose une continuité spatio-temporelle entre les lieux de résidence, ainsi qu'une certaine unité sociale. «Il y a une intégration fonctionnelle des différents lieux entre lesquels les individus circulent. L'intensité des déplacements circulaires peut ainsi modifier la structuration de l'espace, induire un continuum entre le centre urbain et son espace environnant, ou un chevauchement d'espaces par intégration fonctionnelle audelà du continuum physique du bâtì (Dupont, Dureau, 1994 : 807). Introduisant la plurirésidentialité, les migrations circulaires permettent de dépasser la dichotomie classique entre pays de départ et pays d'arrivée, ou entre migration définitive et migration temporaire.

Un autre élément qui apparaît comme inhérent aux migrations circulaires est que l'ensemble du groupe ne pratique pas cette forme de mobilité. Contrairement au nomadisme, qui reprend la circularité ainsi que la répétition des déplacements et implique la mobilité de tous, les migrations circulaires supposent que seule une partie des individus se déplacent, les autres étant cantonnés dans l'un ou l'autre des lieux mis en relation. Ainsi, l'immobilité est partie prenante des migrations circulaires.

Figure $\mathbf{n}^{\circ} 2$ : Des migrations circulaires à la culture de la mobilité.

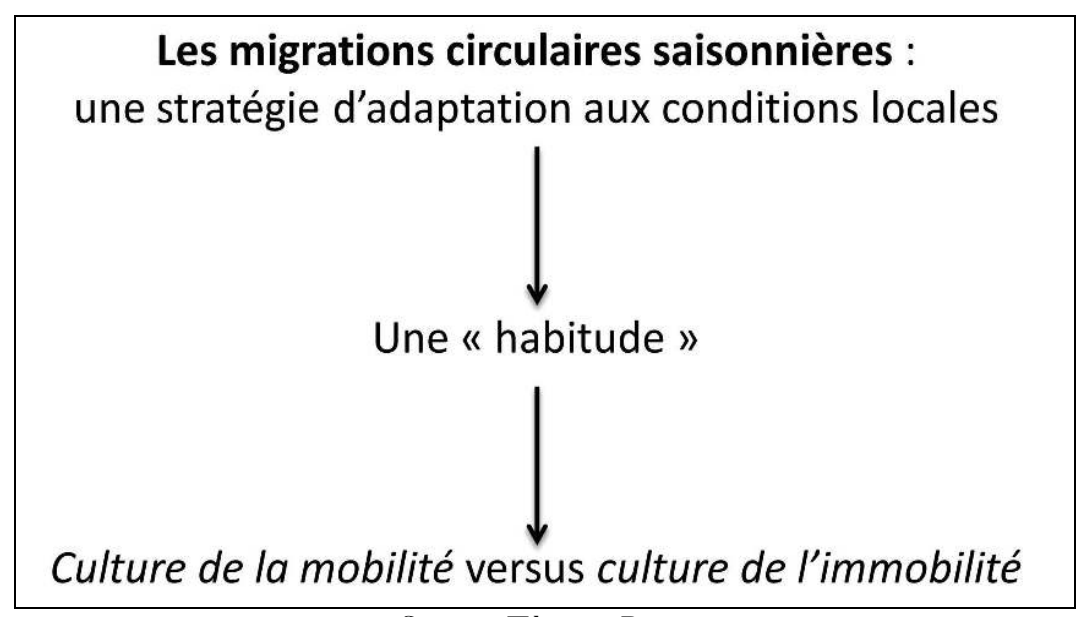

Source : Florence Boyer.

Dans le contexte sahélien, les migrations circulaires contemporaines s'inscrivent dans la continuité de formes de mobilité anciennes, reliant l'espace rural aux espaces urbains. En effet, la colonisation, la monétarisation de l'économie auraient conduit une partie de la population à se rendre temporairement en ville pour accéder à des ressources non disponibles dans l'espace rural (Chapman, Prothero, 1983). Classiquement, les migrants se déplaçaient vers les villes pendant la saison sèche, qui correspond à une période d'inactivité en milieu rural, avant de rentrer participer aux cultures pendant la saison des pluies. Longtemps présentées comme une stratégie d'adaptation aux conditions climatiques et environnementales, ces migrations sont 
aujourd'hui devenues une habitude (terme employé de façon récurrente par les migrants eux-mêmes pour expliquer leur départ), au point que certains auteurs parlent de «culture de la mobilité » (de Bruijn, van Dijk, Foeken, 2001).

Cependant, si les migrations circulaires sont inscrites dans les pratiques habituelles de nombre de populations sahéliennes, au point d'être devenues une norme, elles ne peuvent se réaliser qu'à la condition qu'une partie de cette population reste sur place, soit stable, autant au niveau spatial que temporel. À cette "culture de la mobilité » serait ainsi associée une «culture de l'immobilité ».

Carte $\mathbf{n}^{\circ} 1$ : Localisation des villages enquêtés, départements de Tahoua, Keita, Illéla et Bouza.

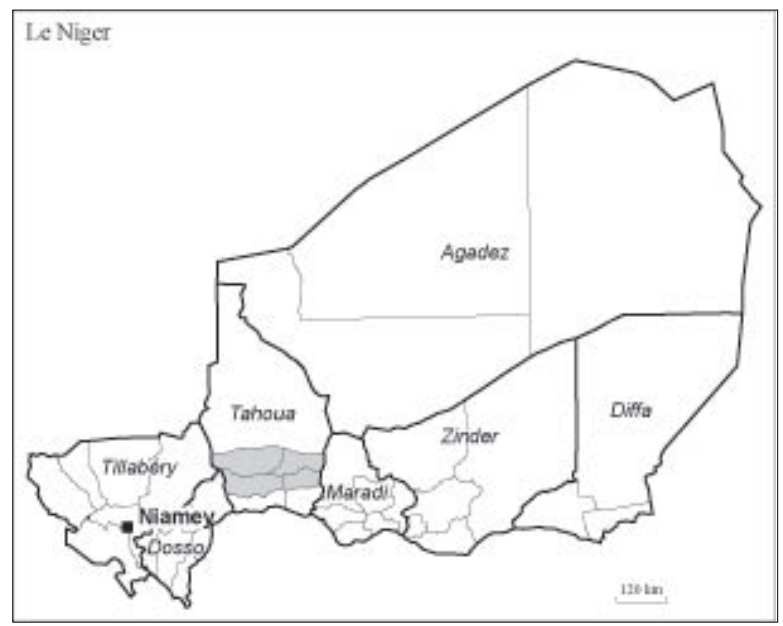

Carte $n^{\circ} 1$ : Florence Boyer.

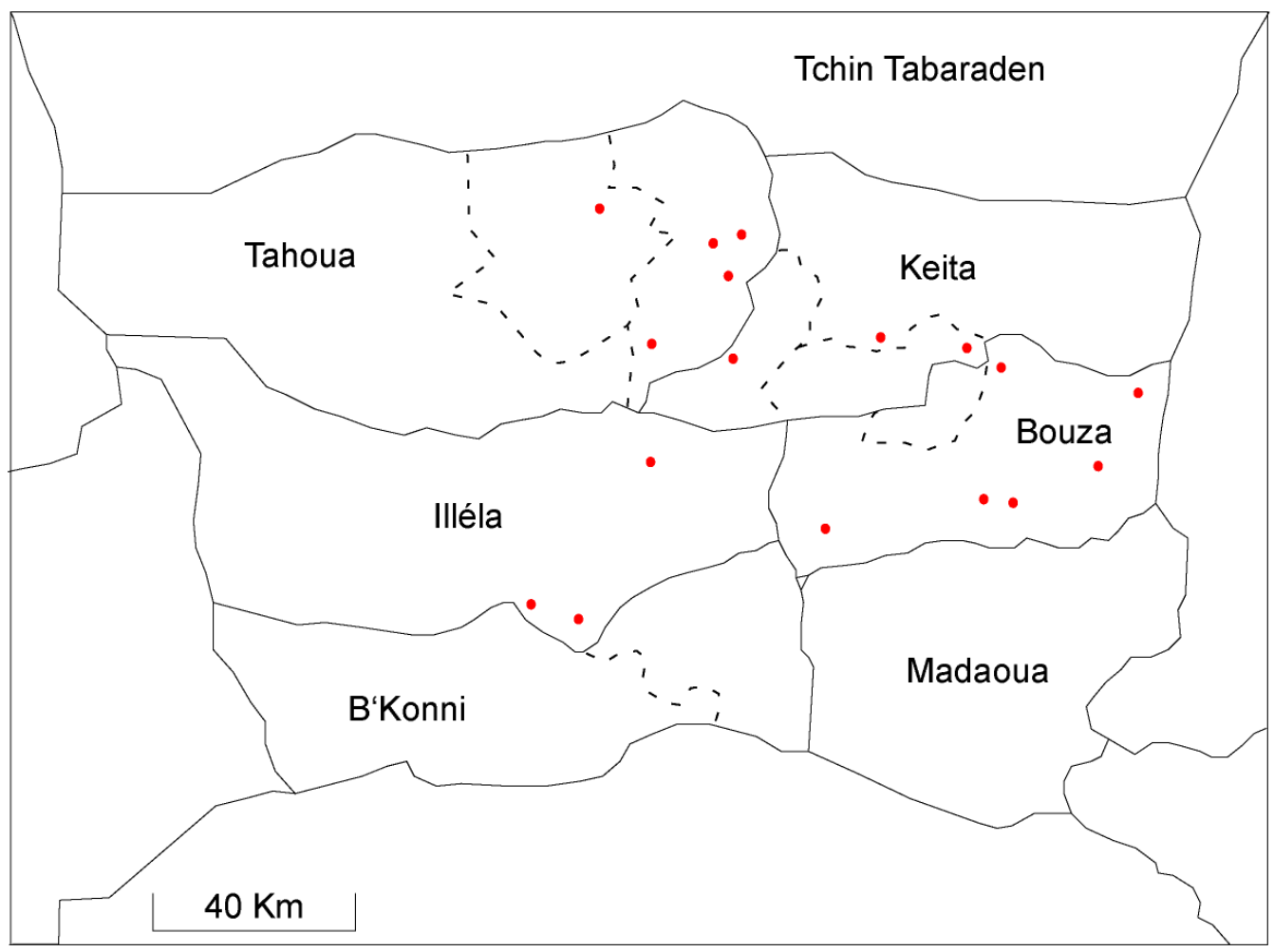

Source: Florence Boyer. 
Située au centre du Niger, dans le massif de l'Ader, la région de Tahoua est connue pour être une importante région de départ à l'échelle du pays; les migrations circulaires s'appuient sur des filières migratoires anciennes, bénéficiant d'installations tout aussi anciennes de membres des groupes sociaux dans les principales villes ouest-africaines. Située également dans la partie nord du Sahel, elle est marquée par l'incertitude de la pluviométrie et partant des récoltes, pourtant fondamentales dans la mesure où l'on se trouve dans un contexte d'agriculture de subsistance; la culture du mil, base de l'alimentation, est généralement associée à un petit élevage qui constitue plus un système d'épargne qu'une véritable ressource. Les villages des vallées peuvent bénéficier des cultures de contre-saison, la culture de l'oignon surtout, seule source de revenu possible de l'agriculture.

Portant sur la partie centrée sur le massif de l'Ader de la région de Tahoua, l'enquête, réalisée en 2008, a concerné 18 villages et hameaux, soit 1145 individus. Associant un volet quantitatif et un volet qualitatif, le protocole d'enquête (Mounkaila, Amadou, Boyer, 2009) avait pour objectif d'apporter une mesure de l'ensemble des mobilités, à différentes échelles spatiales et

Figure $\mathbf{n}^{\circ} \mathbf{3}$ : Système de production agricole et rythme des migrations circulaires.

\begin{tabular}{|l|l|l|}
\hline Typologie des fermes & Destinations des migrations & Rythme des migrations \\
\hline $\begin{array}{l}\text { Culture de mil } \\
\text { uniquement }\end{array}$ & Niamey & $\begin{array}{l}-7 \% \text { de la population absente toute } \\
\text { l'année } \\
- \text { Saisonnalité des mouvements }\end{array}$ \\
\hline Culture de mil et élevage & Afrique de l'Ouest & $\begin{array}{l}-5 \% \text { de la population absente toute } \\
\text { l'année } \\
- \text { Saisonnalité des mouvements }\end{array}$ \\
\hline $\begin{array}{l}\text { Culture de mil et culture } \\
\text { de contre-saison }\end{array}$ & $\begin{array}{l}\text { Afrique de l'Ouest } \\
\text { Afrique du Nord }\end{array}$ & $\begin{array}{l}-10 \% \text { de la population absente toute } \\
\text { l'année } \\
\text {-Disparition de la saisonnalité des } \\
\text { mouvements }\end{array}$ \\
\hline
\end{tabular}

Source : enquête « Mesure des mobilités spatiales. Région de Tahoua », 2008.

temporelles ${ }^{1}$, tout en interrogeant le projet migratoire des individus, ainsi que les liens entre migrants et non-migrants.

Au cours des dernières décennies, le principal constat observé en termes de changement dans la pratique des migrations circulaires est l'évolution de leur rythme. Comme les hommes les plus âgés en font souvent la remarque au cours des entretiens, les migrants sont absents de plus en plus

\footnotetext{
${ }^{1}$ Ainsi les mobilités ont été mesurées à l'échelle de l'année précédant l'enquête, de la vie de l'individu (approche biographique) et du quotidien.
}

longtemps; dépassant la seule saison sèche, leur absence peut parfois durer plusieurs années. Une analyse des rythmes sur l'année va dans le même sens que ce constat; en effet, une partie de la population, surtout masculine est absente toute l'année, son importance variant en fonction des systèmes de production locaux, de même que les destinations. Le tableau ${ }^{2}$ indique la part de la population absente depuis au moins une année au moment de l'enquête, en fonction

\footnotetext{
${ }^{2}$ L'ensemble des traitements statistiques a été réalisé par Guillaume Le Roux (Doctorant, UMR 7301, Migrinter)
} 
du système de production des exploitations agricoles et des principales destinations des migrants.

Un tel constat a plusieurs conséquences, notamment pour les immobiles, ceux qui ne pratiquent pas ou plus ces migrations. D'une part, l'atténuation, voire la disparition du caractère saisonnier de ces mouvements, amène au fait que les personnes mobiles ne peuvent plus participer aux activités villageoises, en particulier à l'agriculture. Ainsi, certaines catégories, qui n'étaient auparavant pas concernées par les travaux des champs, se trouvent aujourd'hui contraintes d'y participer; il s'agit notamment des femmes ou des jeunes enfants. D'autre part, l'allongement des durées d'absence entraîne des spécialisations en fonction des individus; alors que certains sont amenés à migrer et par conséquent à exercer une activité hors du village, d'autres restent dans ces mêmes villages ou ne partent que pendant la saison sèche. Cependant, tous participent au maintien de la population villageoise sur place, comme nous le verrons par la suite.

Figure $\mathbf{n}^{\circ} 4$ : Distribution des âges selon les principaux événements de la vie.

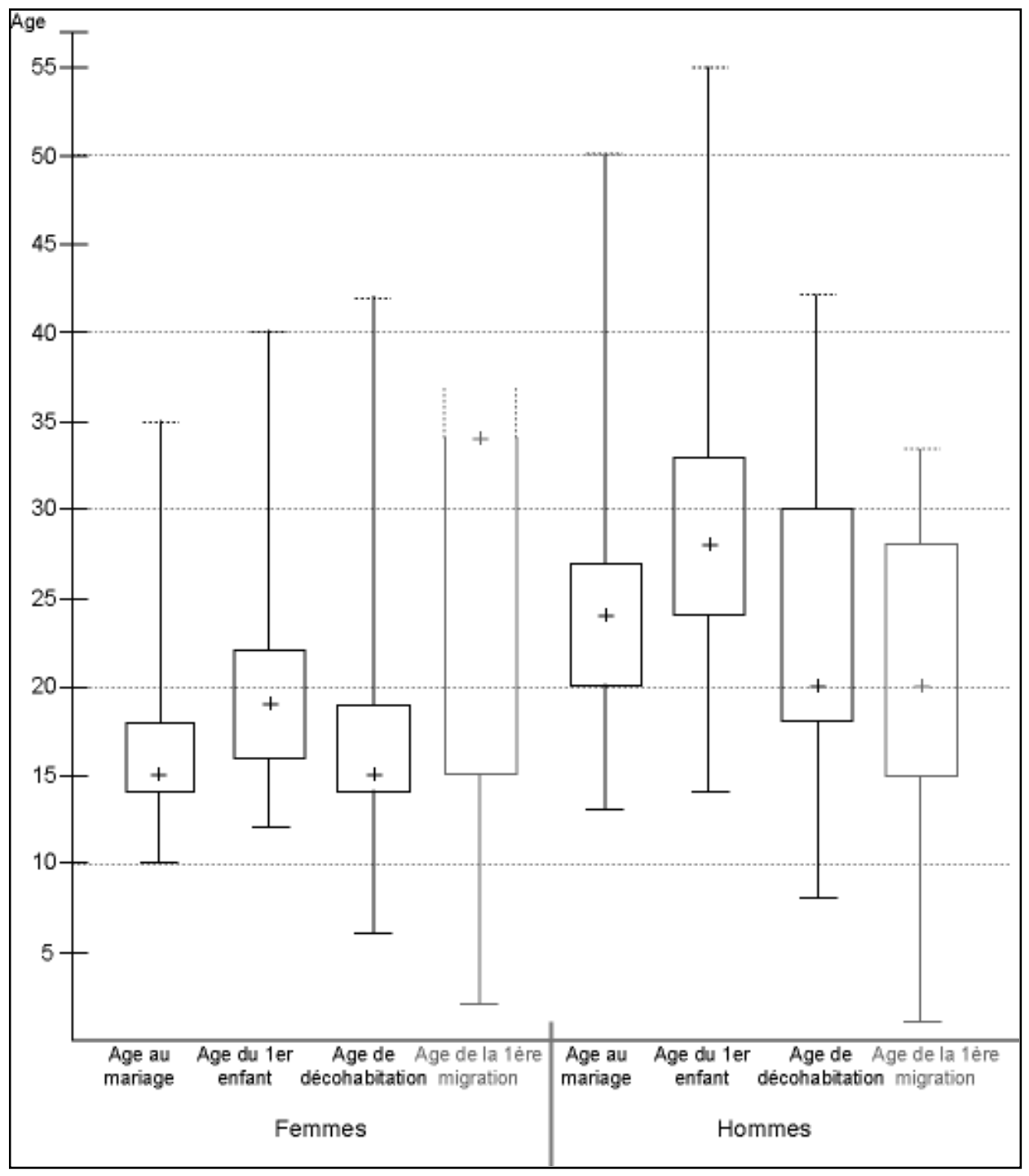

Source : enquête «Mesure des mobilités spatiales. Région de Tahoua », 2008. 
Afin de mieux comprendre qui sont les individus appelés à migrer ou non, il est possible de se concentrer sur les principaux événements vitaux de chacun, en distinguant la population selon le genre. Ainsi, sont observés l'âge au mariage, l'âge à la naissance du premier enfant, l'âge de la décohabitation ainsi que celui de la première migration. Un décalage important entre les hommes et les femmes s'observe pour l'ensemble de ces événements vitaux. Alors que l'âge médian des femmes au mariage est de 15 ans, il est de 24 ans pour les hommes; cependant, cet indicateur est à prendre avec précaution, dans la mesure où le mariage peut avoir lieu avant le paiement de la dot, ce qui signifie qu'il ne sera consommé qu'au versement de celle-ci, même si l'épouse emménage dans la cour de son mari. L'âge médian du premier enfant confirme ce fonctionnement, dans la mesure où il passe à 19 ans pour les femmes et 28 ans pour les hommes. Toujours pour ce qui est de la population féminine, on observe une concordance entre l'âge au mariage et la décohabitation, mais un décalage pour ce qui est de la migration. Dans la mesure où par migration, il est entendu changement de village, il apparait que les femmes non seulement ne migrent pas, mais se marient à l'intérieur de leur village d'origine.

Pour les hommes la situation est assez différente; leur premier événement de la vie est la migration, qui survient relativement tôt, puisque l'âge médian de la première migration est de 20 ans et le quart de la population masculine a migré avant 15 ans. Les départs précoces (au moment de l'adolescence) des hommes en migration de travail sont confirmés autant par l'analyse des trajectoires biographiques que par les entretiens. L'observation de l'intensité de la migration tout au long de la vie de l'individu montre qu'entre 15 et 30 ans, quasiment tous les hommes ont migré au moins une fois ; une première diminution de l'intensité de migration a lieu autour de 35 ans, mais la baisse ne s'amorce vraiment qu'autour de 45 ans. Ainsi, il apparaît que les migrations circulaires sont avant tout masculines; ne restent dans les villages que les femmes, les hommes les plus âgés et les jeunes enfants. Parmi ces trois catégories, seules les femmes sont présentes aux âges actifs.

\section{De la dépendance des immobiles face aux migrants circulaires}

Figure $\mathbf{n}^{\circ} \mathbf{5}$ : Projets migratoires : initiative individuelle et contrat migratoire.

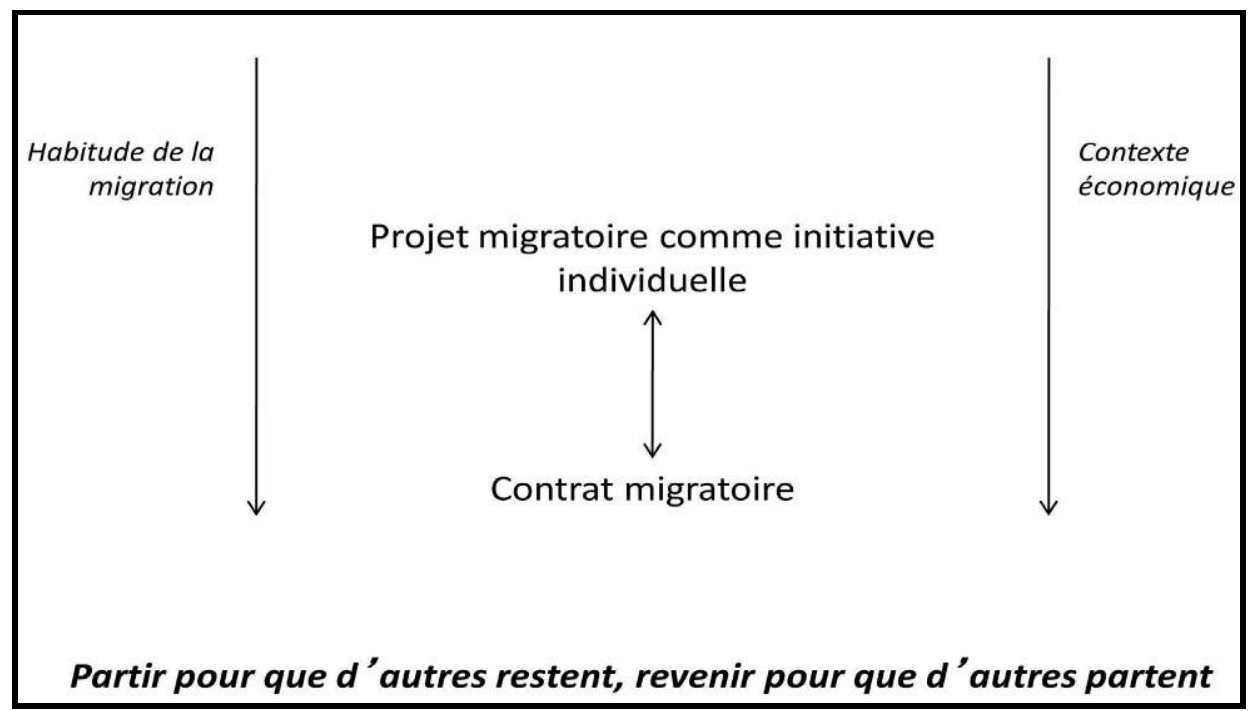

Source : Florence Boyer. 
La pratique des migrations circulaires par les hommes d'âge actif, pourrait se résumer ainsi : ils partent, permettant à d'autres de rester; ils reviennent, permettant à d'autres de partir. D'une certaine manière, alors que la migration se transmet chez les hommes au fil des générations, l'immobilité se transmet tout autant chez les femmes. Si l'habitude de migrer, comme les nécessités économiques s'envisagent comme autant de facteurs concourant aux départs, les mouvements sont également portés par deux autres éléments indissociables dans ce cas, le projet migratoire d'une part et le contrat migratoire d'autre part. Alors que le projet migratoire renvoie à une dimension individuelle (certes articulée à une dimension collective), le contrat migratoire fait plus directement référence à une dimension collective. Contrat tacite, non formulé, mais qui pèse sur les comportements des uns et des autres : les jeunes hommes pratiquent les migrations circulaires, les femmes sont cantonnées dans les villages sous la "surveillance» des hommes plus âgés. Cependant, les premiers se doivent de subvenir aux besoins des seconds et notamment de pallier les manques de nourriture, résultant de la déficience structurelle des récoltes.

Photographie $\mathbf{n}^{\circ} \mathbf{3}$ : Télécentre à Tahoua (mode d'envoi d’argent informel) : liste des numéros de téléphone de personnes résidant dans les villages à contacter lors de la réception d'un transfert d'argent.

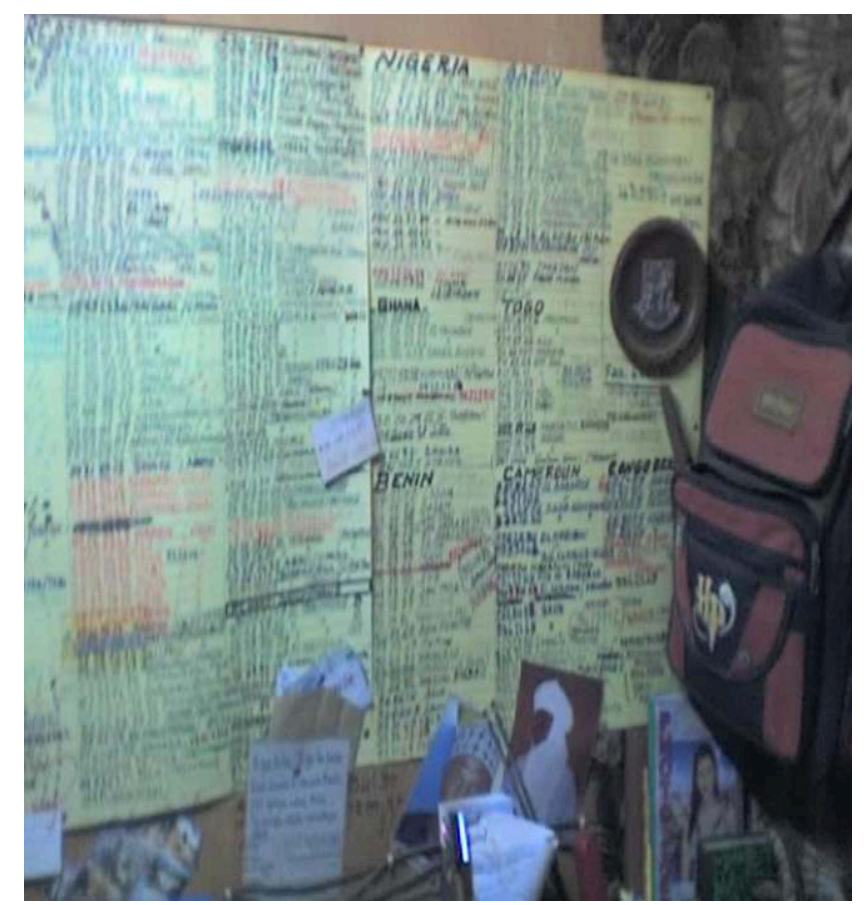

Source : photo d'Abdoul-Razak Garba.

La situation «d'habitude de la mobilité » actuelle qui se conjugue avec une situation «d'habitude de l'immobilité» s'appuie certes sur un système de complémentarité, mais surtout sur un système d'aide, dont la manifestation principale est l'envoi de fonds des migrants, ou l'argent et les biens qu'ils ramènent à leur retour. Pourtant importante région de départ, Tahoua est aussi une région où la migration ne se voit pas; hormis quelques mosquées ou villas, surtout construites par des grands commerçants installés à Niamey et originaires de la région, les villages ne présentent pas de signe visible d'investissements importants. Deux éléments peuvent expliquer cette situation: d'une part, les ressources des migrations circulaires restent modestes, d'autre part la question de la sécurité alimentaire est telle 
que l'essentiel de ces ressources sert à nourrir les membres de la famille restés au village. Si une enquête auprès de deux banques (dont l'une reçoit les mandats de Western Union) et des lieux de réception informels des remises à Tahoua a montré que plusieurs millions de francs CFA arrivent quotidiennement dans cette ville en provenance des grandes capitales de l'Afrique de l'Ouest ou de la Libye, il s'avère que cette somme est dispersée entre une multitude de familles, comme en témoigne la longue liste de numéros de téléphone sur la photo. En effet, les migrants de retour affirment n'envoyer que quelques milliers de francs CFA chaque mois à leur famille lorsqu'ils envoient quelque chose - et/ou ne rentrer qu'avec quelques milliers, voire centaines de milliers de francs CFA. Toutefois, si les revenus de la migration restent modestes, ils parviennent non seulement à subvenir aux besoins en nourriture, mais aussi aux besoins en biens de consommation courante. Un tel système aboutit à une situation de dépendance réciproque.

Figure $\mathbf{n}^{\circ} \mathbf{6}$ : Une relation de dépendance sous contrôle.

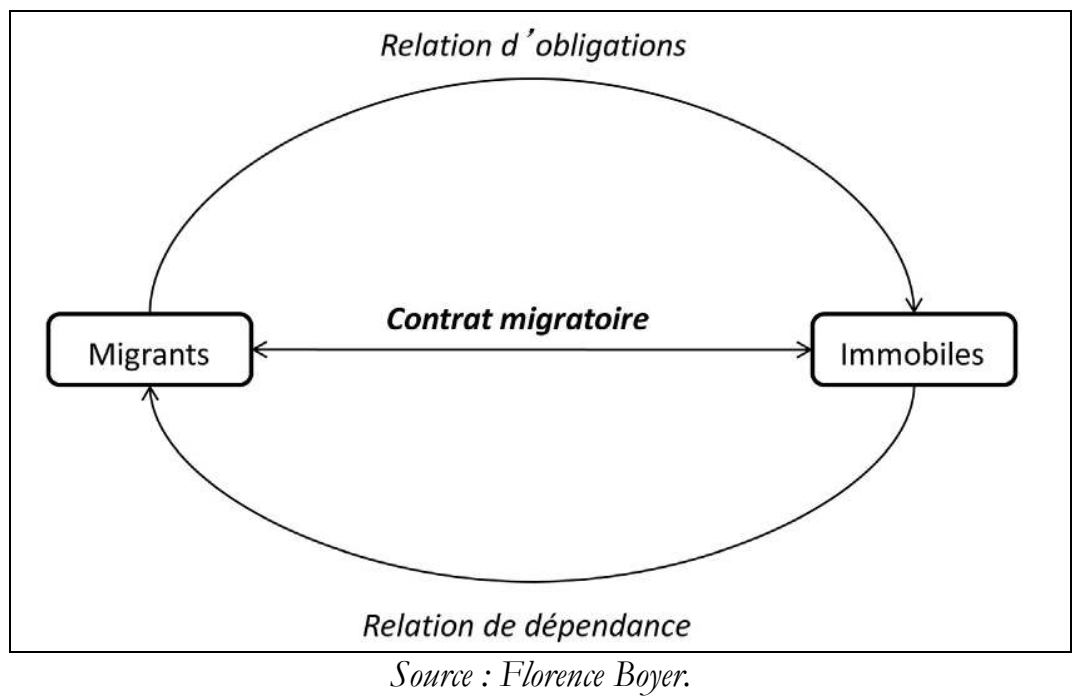

Le contrat migratoire dont la manifestation la plus concrète, et quasiment vitale, correspond aux remises de fonds des migrants, s'inscrit dans une relation de dépendance doublée d'une relation d'obligations. Alors que les immobiles dépendent en partie de la mobilité pour leur survie, les migrants sont dans l'obligation, certes de partir, mais surtout de revenir, n'ayant pas véritablement le choix de s'installer à l'extérieur et d'y faire venir leur famille. En effet, le contrat migratoire est aussi à inscrire dans le cadre des hiérarchies sociales et d'une organisation familiale contrôlée par les aînés, eux-mêmes anciens migrants. En transmettant la pratique des migrations circulaires à leurs cadets, ils transmettent également l'ensemble des obligations qui vont avec; ils ont permis à leurs cadets de rester dans les campements, d'être immobiles pendant un temps. À leur tour ceux-ci autorisent l'immobilité en "partant chercher», pour reprendre une expression très usitée dans les campements. Le contrôle social exercé par les plus âgés, autant sur les jeunes hommes que sur les femmes est le garant de la pérennité d'un tel système.

\section{Le point de vue des immobiles? La figure de Pénélope}

Jusqu'à présent nous avons décrit la complémentarité, les liens entre les mobiles et les immobiles, du point de vue des premiers, c'est-à-dire que nous avons décrit les migrations circulaires, leur 
fonctionnement et leur rôle dans l'espace local. Il est possible d'inverser le regard pour adopter le point de vue des immobiles. Une telle perspective amène à recueillir la parole de ceux qui ne se déplacent pas, ici les personnes âgées et les femmes. Cependant, ce renversement, en ce qu'il est inhabituel, se heurte à des difficultés. La première, et la plus importante, est que l'objet de cette recherche était les migrations circulaires; aussi cette parole a été laissée de côté pour privilégier celle des migrants. Rares sont les femmes avec lesquelles nous nous sommes entretenus dans la mesure où elles n'entraient pas dans notre questionnement. La seconde est qu'il est difficile de s'entretenir avec ces mêmes femmes, notamment parce que leur parole, leurs gestes sont contrôlés par les hommes, qu'ils soient maris, pères ou beaux-pères. Cloîtrées dans quelques villages, elles n'ont pu intervenir dans les discussions collectives ou les entretiens qui ont lieu. Si certaines ont répondu au questionnaire, celui-ci n'est qu'un pâle reflet de leur point de vue autant sur les mobilités que sur leur immobilité. Si des outils auraient pu être mis en place pour contourner le contrôle social, cela n'a pas eu lieu, une fois encore parce que l'objet de la recherche était les migrations. Aussi, le seul point de vue des immobiles recueilli est celui d'anciens migrants, à savoir les hommes les plus âgés, qui ont cessé leurs déplacements, remplacés par leurs fils ou leurs frères.

Figure $\mathbf{n}^{\circ} \mathbf{7}$ : Adopter le point de vue des immobiles, quels difficultés?

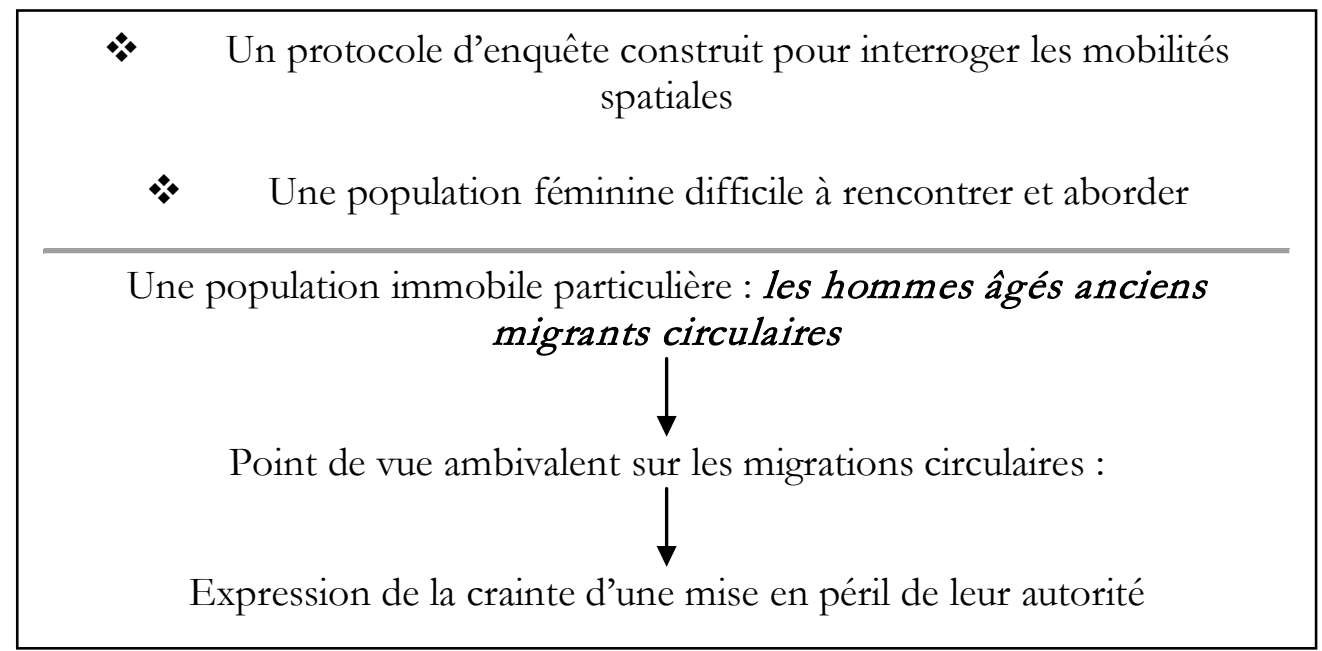

Source : Florence Boyer, 2007.

Les hommes les plus âgés ont un discours paradoxal sur les mobilités. Euxmêmes anciens migrants, ils reconnaissent la nécessité de la pratique migratoire, dans la mesure où elle apporte une aide indispensable au quotidien dans les villages. Cependant, ce premier niveau de discours se double d'un second, plus critique. Sont pointés du doigt les migrants actuels, non parce qu'ils partent, mais parce qu'ils partent plus longtemps que leurs aînés. L'allongement des durées d'absence est mis en cause, interprété comme un non-respect du contrat migratoire. En effet, les durées d'absence grandissantes constituent un risque pour les immobiles, dans la mesure où elles peuvent déboucher sur une installation hors du village. Également, elles sont un risque pour les systèmes de production locaux; dans certains villages, les plus âgés ont fait état d'une réduction des surfaces mises en culture en raison du manque de main d'œuvre, les femmes et les enfants n'ayant pas la capacité de remplacer les jeunes hommes. 
Photographie $\mathbf{n}^{\circ} 5$ : La figure de Pénélope : attente et solitude des femmes (lieu : Nagaro).

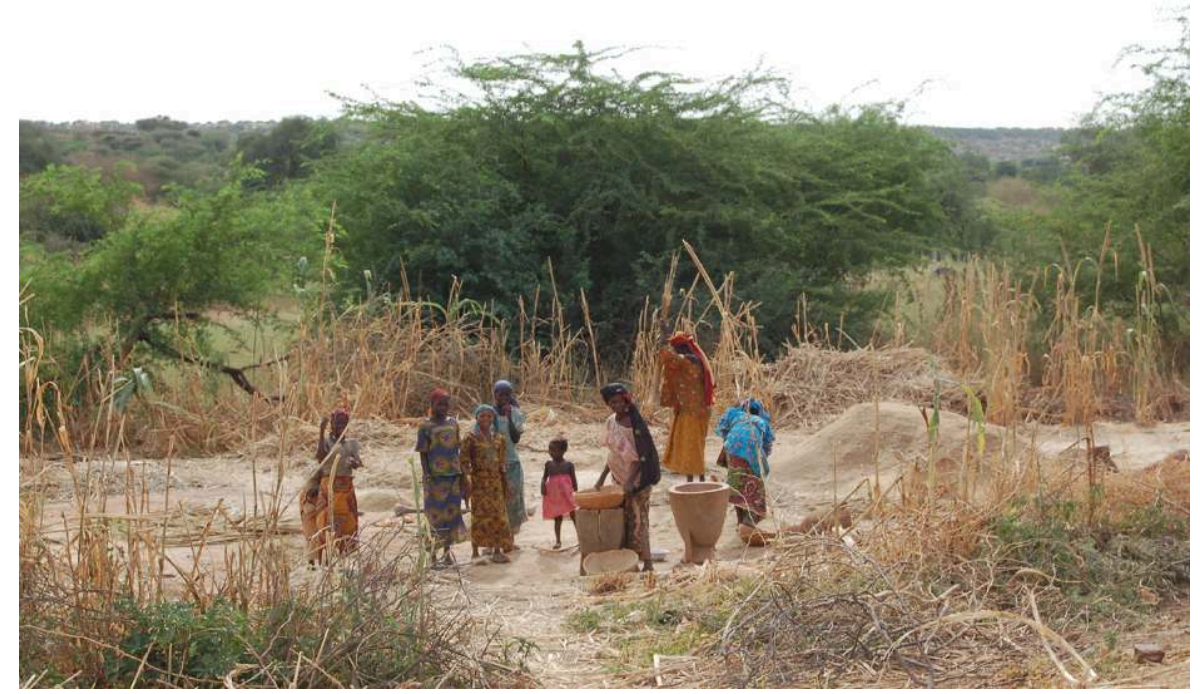

Source : Florence Boyer, 2007.

Bien que nous ne disposions que de peu d'informations directes quant au point de vue des femmes sur la mobilité et leur immobilité, il est possible de faire ressortir des éléments, permettant de qualifier leur position. Les femmes sont dans une double position d'attente; d'une part, elles attendent le retour de leur mari (ou éventuellement de leurs frères ou père), d'autre part, elles sont maintenues dans cette position par les aînés qui exercent un contrôle social. Ainsi, malgré l'absence de leur époux, les femmes restent dans la famille de leurs beaux-parents, constituant ainsi une main d'œuvre à disposition pour les tâches ménagères et parfois pour les cultures ou l'entretien des troupeaux. Par les travaux qu'elles effectuent, elles ont alors à leur charge leurs beaux-parents, souvent trop âgés pour gérer le quotidien. Nous avons repris la figure de Pénélope qui non seulement attend le retour d'Ulysse, mais doit contourner l'autorité de ses ânnés pour ne pas être mariée à l'un des prétendants, pour qualifier la situation de ces femmes.

Empêchées de suivre leur conjoint en migration, sauf dans de très rares cas, par les plus âgés, l'immobilité contrainte des femmes fait qu'elles se retrouvent avec de fortes charges pendant plusieurs mois, voire plusieurs années, sachant que leur mari n'envoie pas forcément régulièrement de l'argent. L'attente place souvent les femmes dans une situation très précaire: certaines doivent cultiver le champ familial ${ }^{3}$, demandant alors la participation de leurs enfants, y compris à des âges très jeunes, ce qui les empêche d'être scolarisés dans des conditions normales. La seule échappatoire pour ces femmes livrées à l'attente, et son corollaire ici la solitude, est que si elles restent deux ans sans nouvelle de leur mari, elles ont la possibilité de demander le divorce, celui-ci pouvant être prononcé sans l'accord de ce même mari.

Ainsi, la contrainte de l'immobilité imposée aux femmes se double d'une autre contrainte, celle de l'obéissance aux logiques de la hiérarchie sociale.

\footnotetext{
${ }^{3}$ Effectués à la main, les travaux des champs sont particulièrement pénibles, notamment les sarclages. Dans la tradition, les femmes ne participent qu'éventuellement au semis, plaçant simplement les graines dans les poquets; elles récoltent également une partie du champ familial (ce qui correspond à leur part). Le fait que certaines soient contraintes d'effectuer l'ensemble des travaux de culture va donc à l'encontre des rôles et travaux dévolus habituellement aux femmes.
} 


\section{Masculinité du pouvoir et gérontocratie : \\ les garants de la stabilité et de la reproduction du système.}

Les migrations circulaires constituent ainsi une forme de mobilité, qui plus que d'autres, implique l'immobilité d'une partie du groupe. Comme nous l'avons déjà signalé précédemment, certains partent pour que d'autres restent, tous rentrent pour que d'autres partent. Ainsi au fil des générations, le système de mobilité se reproduit, de même que celui de l'immobilité, les plus âgés se présentant à la fois comme les garants du système et de sa reproduction. Cependant, la transmission des pratiques spatiales de génération en génération ne s'effectue que parce que la hiérarchie sociale est transmise. Anciens migrants, les plus âgés conservent l'autorité qu'ils transmettront ensuite aux migrants actuels. Masculinité et gérontocratie se conjuguent pour assurer la pérennité du système et des pratiques spatiales afférentes. Ainsi, les seules migrations circulaires ne pourraient maintenir la stabilité de la population des villages; le contrôle social, de même que sa reproduction, est le garant de cet équilibre, certes fragile, entre mobiles et immobiles.

Florence Boyer

CR IRD

UMR 205 Migrations et Société (URMIS) florence.boyer@ird.fr

\section{Bibliographie $^{4}$}

Barrère, Anne; Martuccelli, Danilo (2005) La modernité et l'imaginaire de la mobilité : inflexion contemporaine, Cabiers Internationaux de Sociologie, ${ }^{\circ} 118$, pp. 55-79.

${ }^{4}$ La bibliographie renvoie à des citations dans le texte, ainsi qu'à des références qui ont nourri la réflexion, sans être pour autant citées.
Bacqué, Marie-Hélène ; Fol, Sylvie (2008) L'inégalité face la mobilité : du constat à l'injonction, Revue Suisse de Sociologie, vol. 33, $\mathrm{n}^{\circ} 1$, pp. 89-104.

Boyer, Florence; Mounkaila, Harouna (2010) Partir pour aider ceux qui restent ou la dépendance face aux migrations, Hommes \& Migrations, n¹286-1287, pp. 212-220.

Chapman, Murray; Prothero, Mansell R. (1983) Themes on circulation in the Third World, International Migration Review, vol. 17, $\mathrm{n}^{\circ} 4$, pp. 597-632.

De Bruijn, Mirjam ; van Dijk, Rijk ; Foeken Dick (2001) Mobile Africa. Changing patterns of movement in Africa and beyond, Leiden, Brill, $211 \mathrm{p}$.

Dupont, Véronique ; Dureau, Françoise (1994) Rôle des mobilités circulaires dans les dynamiques urbaines. Illustrations à partir de l'Equateur et de l'Inde, Revue Tiers Monde, vol. 35, n¹40, pp. 801-829.

Dureau, Françoise; Florez, Carmen Elisa (2000) Aguaitacaminos. Las transformaciones de las ciudades de Yopal, Aguazul y Tauramena durante la explotocion petrolera de CusianaCupiagua, Santafé de Bogota, Ediciones Uniandes, 364 p. (Tercer Mundo).

Fol, Sylvie (2010) Encouragement ou injonction à la mobilité ?, Projet, $\mathrm{n}^{\circ} 314$, pp. 52-58.

Mounkaila, Harouna ; Amadou, Boureima ; Boyer, Florence (2009) Le Niger, espace d'émigration et de transit vers le sud et le nord du Sahara : rôle et comportement des acteurs, recompositions spatiales et transformations socio-économiques, in Document de synthèse des projets du programme FSP 2003-74: migrations internationales, recompositions territoriales et développement, Paris, IRD, pp. 109-120. 